\title{
Edema pulmonar agudo por uso de tiazidas, ¿existe falla cardíaca? Comunicación de un caso
}

\author{
Max Andresen $\mathrm{H}$, Alejandro González $\mathrm{M}^{\mathrm{b}}$, Alberto Espino $\mathrm{E}^{\mathrm{a}}$ \\ Marcelo Mercado F, Tomas Regueira H, Alberto D ougnac $L$.

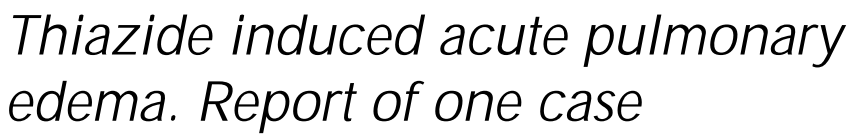

Acute pulmonary edema caused by thiazides is uncommon and of difficult diagnosis. It is considered an idiosyncratic reaction and the physiopathology or cardiac function changes are not well known. We report a 60 year-old female with a thiazide induced acute pulmonary edema who was followed with serial measurements of type B n-terminal natriuretic peptide fraction as marker for cardiac dysfunction. There was a significant elevation of the peptide, not associated to evidences of ventricular dysfunction. Its normalization paralleled the resolution of the clinical picture (Rev Méd Chile 2007; 135: 496-500).

(Key words: Natriuretic peptide type B; Pulmonary edema; Thiazides)

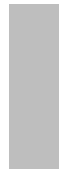

\footnotetext{
Recibido el 16 de noviembre, 2005. Aceptado el 19 de julio, 2006.

Programa de Medicina Intensiva, Unidad de Tratamiento Intensivo Médico, Facultad de Medicina, Hospital Clínico Pontificia Universidad Católica de Chile.

aesidente de Medicina Interna

bInterno de Medicina
}

$\mathrm{U}$ na de las causas frecuentes de insuficiencia respiratoria aguda que requieren ingreso a una Unidad de Cuidados Intensivos (UCI), es el edema pulmonar agudo (EPA). Este puede clasificarse como de origen cardiogénico, por aumento de la presión hidrostática vascular o no cardiogénico, secundario a un aumento de la permeabilidad vascular pulmonar.

El síndrome de distress respiratorio agudo (SDRA) es considerado como una forma no cardiogénica de edema pulmonar. La conferencia

Correspondencia a: Dr. Max Andresen H. Unidad de Tratamiento Intensivo Médico, Hospital Clínico Pontificia Universidad Católica de Chile. Marcoleta 367, Santiago. E mail: andresen@med.puc.cl de consenso americana-europea lo define como un cuadro de insuficiencia respiratoria de inicio agudo, que cursa con una $\mathrm{PaO} 2 / \mathrm{FIO} 2 \leq 200$ $\mathrm{mmHg}$, en presencia de una radiografía de tórax con infiltrados bilaterales difusos y una presión de capilar pulmonar (PCP) $\leq 18 \mathrm{mmHg}$ o ausencia de hipertensión auricular izquierda. Una forma más moderada de SDRA es el ALI (del inglés acute lung injury) definido por una relación PaO2/FIO2 $\leq 300 \mathrm{mmHg})^{1}$.

Entre los principales factores de riesgo para desarmollar esta patología están las infecciones, trauma y la pancreatitis. El desarmollo de SDRA asociado a drogas es infrecuente correspondiendo sólo a 3\% de los casos $^{2}$. Numerosos fármacos se han asociado al desarrollo de EPA no cardiogénico, pero sólo pocas veces se puede establecer relación causal ${ }^{3}$, considerando lo inespecífico del cuadro clínico. 
El EPA no cardiogénico inducido por hidroclorotiazida es una entidad poco frecuente. Desde que fue descrito por primera vez por Steinbergen en $1968^{4}$, el número de casos reportados asciende en la actualidad sólo a 36 pacientes, seis de ellos comunicados en esta revista compatibles con este cuadro, el último en $1996^{5}$. Hasta esta fecha existe un escaso conocimiento de la fisiopatología del cuadro clínico, planteándose incluso, en algunos pacientes, disfunción cardíaca asociada ${ }^{6}$.

El péptido natriurético cerebral (BNP) y la fracción N Terminal del pro-BNP (NT-pro-BNP) han aparecido como excelentes indicadores biohumorales de disfunción de ventrículo izquier$\mathrm{do}^{7,8}$. Presentamos el caso de una paciente que desarrolló cuadro de edema agudo de pulmón secundario a tiazidas, que presentó elevación inicial del NT-pro-BNP en ausencia de falla ventricular izquierda demostrable por diferentes métodos, hecho que se contrasta con otro reporte de la literatura internacional ${ }^{6}$.

Se discute este interesante caso a la luz de nuevos elementos descritos en su patogenia.

\section{CASO CĹNICO}

Mujer de 60 años, con antecedentes de embolia pulmonar a repetición, tratamiento anticoagulante oral suspendido desde un año antes, filtro de vena cava inferior instalado 11 meses antes, hipertensión arterial y obesidad mórbida. Usuaria de aspirina, omeprazol, clonazepam, citalopram y atenolol, el cual fue reemplazado un día previo al cuadro actual por losartán asociado a hidroclorotiazida. Como antecedente destacaba que en el año 2002, en relación con una ingesta única de hidroclorotiazida, presentó cuadro de disnea súbita y mareos, no bien documentado.

Consultó por cuadro de tres días de evolución caracterizado por tos seca, disnea progresiva, sin dolor torácico, palpitaciones, ni fiebre. $\mathrm{Al}$ ingreso se encontraba polipneica a $30 \mathrm{x}^{\prime}$, cianótica, hipotensa $(80 / 50 \mathrm{mmHg}), \mathrm{FC} 80 \mathrm{x}^{\prime}, \mathrm{T} 36,5^{\circ} \mathrm{C}$, sopor superficial, se decidió intubar y trasladar a UCI.

Examen físico: murmullo pulmonar estaba disminuido en ambos campos con crépitos bilaterales abundantes, el examen cardíaco era normal, sin soplos. El resto del examen no mostró alteraciones significativas.

Se intubó y conectó a ventilación mecánica, los gases arteriales de ingreso con $\mathrm{FiO} 2$ de $100 \%$ mostraban: pH 7,35; pCO2 33,1 mmHg; paO2 85,9 $\mathrm{mmHg}$; HCO3 18,4 mEq/lt; $\mathrm{BE}-5,4 ; \mathrm{PaO} 2 / \mathrm{FiO} 2$ 85,9. Hemograma: hematocrito de $52,5 \%$, un recuento de blancos de $3.900 \mathrm{~mm}^{3}$. El resto de los exámenes de laboratorio no mostraron alteraciones. Electrocardiograma: taquicardia sinusal 120 $\mathrm{x}^{\prime}$, sin evidencia de isquemia, troponina I de ingreso $0,02 \mathrm{ng} / \mathrm{ml}$. Radiografía de tórax compatible con edema pulmonar (Figura 1).

Se instaló catéter de Swan-Ganz, que evidenció gastos cardíacos entre 6 y 7 L/min, PCP siempre menores de $18 \mathrm{mmHg}$, resistencias vascu-

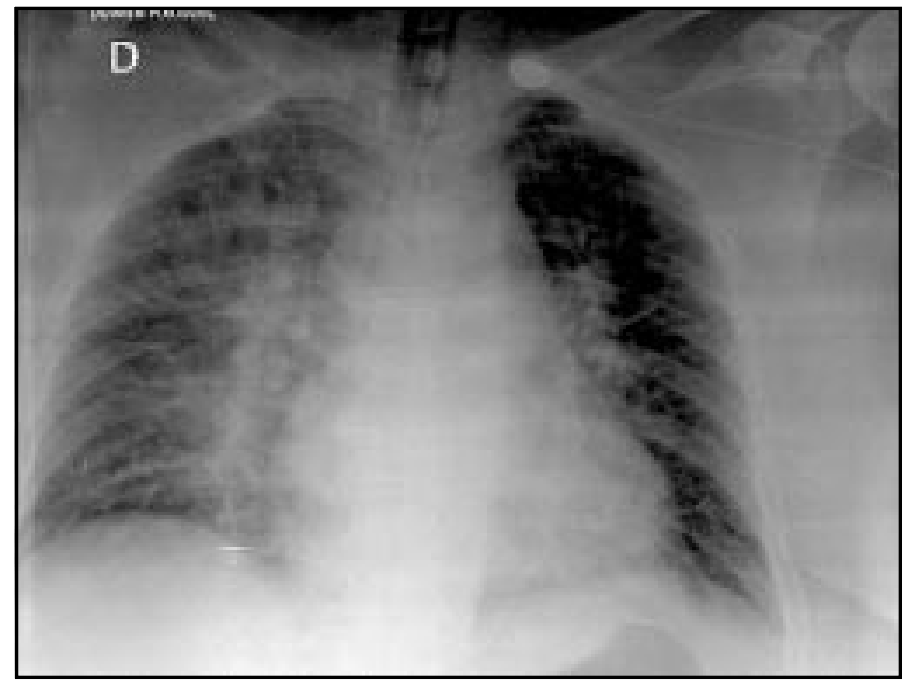

Figura 1. Radiografía de tórax AP portátil al ingreso, muestra tenue velo derecho compatible con derrame pleural de distribución posterior, engrosamiento intersticial peribroncovascular difuso de predominio perihiliar, asociado a líneas septales y áreas de relleno alveolar a derecha, compatible con edema pulmonar. 
lares sistémicas normales y presiones de arteria pulmonar elevadas (Tabla 1). AngioTAC de tórax a las $6 \mathrm{~h}$ del ingreso resultó negativo para TEP (Figura 2).

La paciente evolucionó favorablemente con requerimiento de oxígeno en rápido descenso, se extubó a las 24 h, y se retiró el catéter de SwanGanz a las 36 h. Nueva radiografía de tórax no mostró infiltrados alveolares pulmonares.

Un ecocardiograma a las $24 \mathrm{~h}$ de ingreso mostró una función sistólica conservada con un ventrículo izquierdo no dilatado, con leve disfunción diastólica tipo defecto de la relajación, una presión sistólica de arteria pulmonar (PSAP) de 40 $\mathrm{mmHg}$ y leve insuficiencia tricuspídea.

Las mediciones de NT-pro-BNP (cuyo rango es menor de $222 \mathrm{pg} / \mathrm{ml}$ para mujeres mayores de 50 años) mostraron valores al doble del normal, siendo monitorizados por dos días. Valor de ingreso: $463 \mathrm{pg} / \mathrm{mL}$ y al día siguiente $488,5 \mathrm{pg} / \mathrm{ml}$. Al séptimo día de evolución se recontroló obteniendo valor normal: 74 pg/ml (Figura 3).

\section{DisCUSIÓN}

El daño pulmonar por hidroclorotiazida se presenta en $90 \%$ de los casos en mujeres ${ }^{9}$, mayormente entre la quinta y sexta década. Puede desencadenarse tras la primera dosis de 25 a $50 \mathrm{mg}$, o bien en pacientes que ya habían usado este tratamiento sin presentar síntomas.

En el caso de las recurrencias, la gravedad del cuadro clínico aumenta ${ }^{10,11}$. En nuestra paciente no podemos asegurar que esto sea una recurrencia, ya que no existe documentación fiable del primer episodio, suponemos que fue leve ya que revirtió la sintomotalogía a las pocas horas.

En cuanto a la patogenia, se ha postulado la existencia de un mecanismo inmunológico (reacción antígeno-anticuerpo) que no ha podido ser demostrado. Probablemente se trate de una reacción idiosincrática similar a las descritas por otros fármacos como narcóticos y salicilatos ${ }^{10,11}$. Algunos autores han observado un descenso en la concentración de IgG y complemento lo que hace sospechar un deposito de IgG en la membrana alveolar.

Esto no se produce con el uso de otros diuréticos, ya que cuando estos pacientes han empleado furosemida no han evidenciando reac-
Tabla 1. Parámetros hemodinámicos registrados con Swan-G anz

\begin{tabular}{|lll|}
\hline Variables & Día 1 & Día 2 \\
\hline GC (L/min) & 6,4 & 7,1 \\
IC (L/min/m $\left.{ }^{2}\right)$ & 2,8 & 3,1 \\
PAPS/D (mmHg) & $37 / 20$ & $24 / 13$ \\
RVS (dina/seg/cm $\left.{ }^{5}\right)$ & 748 & 715 \\
PVC (mmHg) & 16 & 6 \\
PAM (mmHg) & 79 & 89 \\
PCP (mmHg) & 17 & 11 \\
SVM (\%) & 67,6 & 74,1 \\
\hline
\end{tabular}

GC: gasto cardíaco, IC: índice cardíaco, PAPS/D: presión de arteria pulmonar sistólica y diastólica, RVS: resistencia vascular sistémica, PVC: presión venosa central, PAM: presión arterial media, PCP: presión de capilar pulmonar, SVM: saturación venosa mixta.

ción cruzada ${ }^{10}$ (nuestra paciente había usado furosemida 10 meses antes sin presentar síntomas adversos).

El cuadro suele ser inespecífico, clásicamente se describe disnea, cianosis, dolor abdominal, náuseas y vómitos. Los síntomas pueden aparecer a los 10 min de ingerir el fármaco. Hasta en un tercio de los casos se describe fiebre autolimitada en las primeras $24 \mathrm{~h}^{12}$ con o sin leucocitosis.

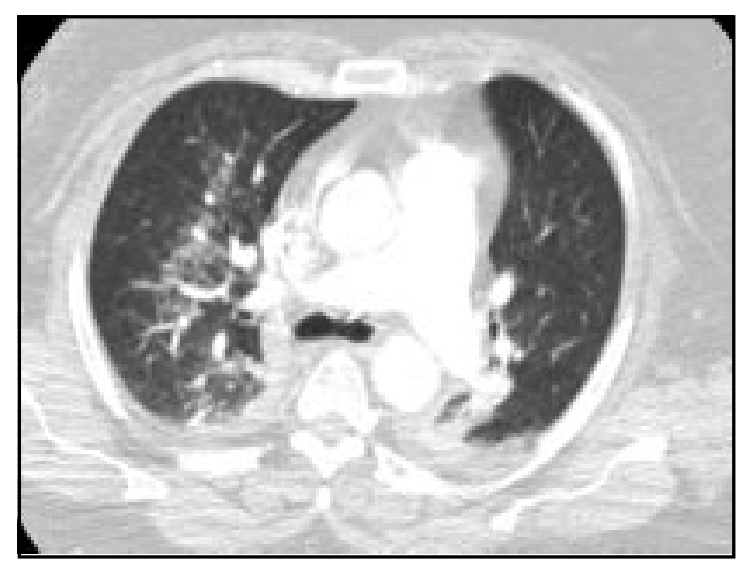

Figura 2. Angio TAC de tórax, examen sin evidencias de TEP agudo. Las opacidades parenquimatosas derechas y el derrame bilateral sugieren cierto grado de insuficiencia cardíaca. Aumento de tamaño de arteria pulmonar central. 


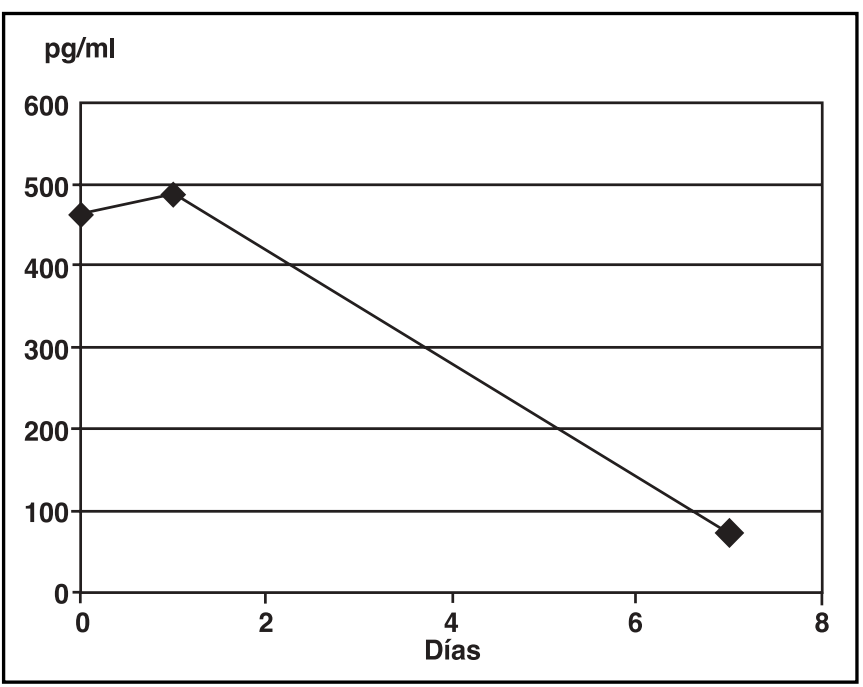

Figura 3. Evolución temporal del NT-pro-BNP.

Los exámenes de sangre frecuentemente son normales, se ha descrito hemoconcentración en pacientes que cursaron con diarrea. Nuestra paciente no cursó con diarrea pero sí hemoconcentración que a las $48 \mathrm{~h}$ se normalizó a un hematocrito de $39,8 \%$. Esto se explicaría por el brusco transudado hacia el espacio alveolar, situación similar a la experimentada por el síndrome pulmonar por Hanta virus $^{13}$. En dos pacientes se ha comunicado leucopenia y trombocitopenia transitoria ${ }^{14}$, esta leucopenia, que también desarrolló nuestra paciente, se cree que es por secuestro intrapulmonar inicial de granulocitos por la estimulación de IL-8.

Se describen dos casos en que se observó depresión reversible de la función miocárdica ${ }^{5,11}$, aunque lo más frecuente es que el monitoreo con Swan-Ganz muestre una presión de enclavamiento normal, lo que indica una función ventricular izquierda conservada. Esta situación se corroboró en nuestra paciente, ya que todas las presiones de capilar pulmonar fueron menores a $18 \mathrm{mmHg}$ y el ecocardiograma descartó disfunción sistólica izquierda.

No existen casos en la literatura que hayan monitorizado NT-pro-BNP en pacientes cursando EPA secundario a tiazidas. Nuestra paciente tuvo niveles elevados (ajustados según edad y sexo), que a los 7 días de evolución bajaron significativamente. El alza inicial no reflejańa disfunción ventricular izquierda, ya que no existió clínica ni estudio hemodinámico consistente con disfunción cardíaca izquierda.

Pensamos que la elevación del NT-pro-BNP se debería, en este caso, a compromiso en la función ventricular derecha, evidenciado por la leve insuficiencia tricuspídea, secundaria a hipertensión pulmonar aguda (objetivada por mediciones directas y por ecocardiograma). Esta se explica por vasoconstricción pulmonar hipóxica y tal vez por liberación de mediadores vasoactivos, hechos habituales en el SDRA ( $\mathrm{PaO} 2 / \mathrm{FiO} 2$ de 85,9 en nuestra paciente, habiéndose descartado TEP). Estudios recientes plantean que este marcador podría incluso no sólo ser secretado a nivel de ventrículo derecho, sino también por el tejido pulmonar ${ }^{15,16}$. En nuestra paciente, no fue posible identificar si el alza del NTpro-BNP fue por daño pulmonar o la falla cardíaca derecha, debido a que ambos estaban presentes.

En la actualidad, el potencial rol para discriminar entre falla cardíaca y SDRA permanece en evaluación. Berman et al mostraron resultados preliminares con niveles de BNP para SDRA significativamente menores en comparación a falla cardíaca $(123 \mathrm{pg} / \mathrm{ml} \mathrm{v} / \mathrm{s} 773 \mathrm{pg} / \mathrm{ml})^{17}$.

Se conoce que con tratamiento de soporte hemodinámico y respiratorio el cuadro clínico evoluciona favorablemente, con resolución de los síntomas en $24 \mathrm{~h}$. En nuestro caso hubo desaparición del patrón de relleno alveolar radiológico en ese periodo, aunque pudiera demorar más días $^{10,18}$ al igual que el aumento en la gradiente alveolo-arterial de oxígeno y la disminución en la capacidad de difusión de monóxido de carbono que puede tomar alrededor de un mes ${ }^{11}$.

Los datos hasta ahora disponibles muestran que sólo un tercio de los pacientes requieren 
ventilación mecánica invasiva con tasas de mortalidad cercanas a $6 \% 19$.

\section{CONCLUSIÓN}

El análisis del caso nos permite decir que no evidenciamos falla del ventrículo izquierdo, a diferencia de lo descrito por Frierson et $\mathrm{al}^{6}$. Lo que podría haber es disfunción del ventrículo derecho, causante del alza del pro-BNP, siendo

\section{REFERENCIAS}

1. Bernard GR, Artigas A, Kenneth L, Brigham KL, Carlet J, FalKe K et al. Report of The AmericanEuropean consensus Conference on Acute Respiratory Distress Syndrome: definitions, mechanisms, relevant outcomes, and clinical trial coordination. Am J Crit Care 1994; 9: 72-81.

2. Rubenfeid G, Caldwell E, Peabody E, Weaver J, Martin D, NeFF M et AL Incidence and Outcomes of Acute Lung Injury. N Engl J Med 2005; 353: 1685-93.

3. REED CR, Glauser FL. Drug-induced noncardiogenic pulmonary edema. Chest 1991; 100: 1120-4.

4. SteinBerg AD. Pulmonary edema following ingestion of hydrochlorothiazide. JAMA 1968; 204: 167-8.

5. Saldaña A, Jalle J, Gaete P, Fajuri A, Castro P, Godoy I ET AL. Edema pulmonar no cardiogénico relacionado con la hidroclorotiazida. Rev Méd Chile 1996; 124: 720.

6. Frierson JH, Marvel SL, Thomas GM. Hydrochlorothiazide -induced- pulmonary edema with severe acute myocardial dysfunction. Clin Cardiol 1995; 18: 112-4.

7. MacLean A, Huang S. The applications of B-type natriuretic peptide measurement in the intensive care unit. Curr Opin Crit Care 2005; 11: 406-12.

8. Bin Yap L, Mukerjee D, Timms PM, Ashrafian H, Coghian JG. Natriuretic Peptides, Respiratory Disease, and the Right Heart. Chest 2004; 126: 1330-6.

9. KNowles SR, Wong GA, Rahim SA, Binkiey K, PhiшPs EJ, Shear NH. Hydrochlorothiazide-induced noncardiogenic pulmonary edema: an underrecognized yet serious adverse drug reaction. Pharmacotherapy 2005; 25: 1258-65.

10. Fine SR, Lodha A, Zoneraich S, Molura JL. Hydro- menos probable la liberación desde el parénquima pulmonar.

Desde el punto de vista clínico, debemos recalcar la necesidad de realizar una exhaustiva anamnesis, incluyendo el uso reciente de fármacos ${ }^{20}$ y conocer sus principales efectos adversos, considerando que en la actualidad es más frecuente ver combinaciones de drogas (asociación de diuréticos más antihipertensivos). El diagnóstico de asociación con la hidroclorotiazida en la primera exposición puede evitar recumencias posteriores con un curso más grave.

chlorothiazide induced acute pulmonary edema. Ann Pharmacother 1995; 29: 701-3.

11. Mas A, Jordana R, Vaués J, Cervantes M. Recurrent hydrochlorothiazide-induced pulmonary edema. Intensive Care Med 1998; 24: 363-5.

12. Bernal C, Pataca R. Hydrochlorothiazide-induced pulmonary edema and associated immunologic changes. Ann Pharmacother 1999; 33: 172-4.

13. Castilo C, Naranjo J, Ossa G. Síndrome cardiopulmonar por hantavirus en 21 adultos en la IX región de Chile. Rev Chil Infectol 2000; 17: 241-7.

14. Prupas HM, Brown D. Acute idiosyncratic reaction to hydrochlorothiazide ingestion. West J Med 1983; 138: 101-2.

15. Bayes-Genis A, Bewdo-Casado J, Zapico E, Cotes C, BELDA J, López L ET AL. N-terminal pro-brain natriuretic peptide reflects pulmonary capillary leakage in patients with acute dyspnea. Am J Cardiol 2004; 94: 669-70.

16. Gerbes A, Dagnino L, Nguyen T, Nemer M. Transcription of brain natriuretic peptide and atrial natriuretic peptide genes in humans tissues. J Clin Endocrinol Metab 1994; 78: 1307-11.

17. Phua J, Keang Lim T, Hoe Lee K. B-type natriuretic peptide: Issues for the intensivist and pulmonologist. Crit Care Med 2005; 33: 2094-103.

18. REED CR, Glauser FL. Drug-induced noncardiogenic pulmonary edema. Chest 1991; 100: 1120-4.

19. Biron P, Dessureault J, NapKe E. Acute allengic interstitial pneumonitis induced by hydrochlorothiazide. Can Med Assoc J 1991; 145: 28-34.

20. Jara Chinarro B, Miguel Diez J, Garcia Satue JL. Edema agudo de pulmón no cardiogénico secundario a hidroclorotiazida. Arch Bronconeumol 2003; 39: 91-3. 